\title{
A Kaiser Window Approach for the Design of Prototype Filters of Cosine Modulated Filterbanks
}

\author{
Yuan-Pei Lin, Member, IEEE, and P. P. Vaidyanathan, Fellow, IEEE
}

\begin{abstract}
The traditional designs for the prototype filters of cosine modulated filterbanks usually involve nonlinear optimizations. We propose limiting the search of the prototype filters to the class of filters obtained using Kaiser windows. The design process is reduced to the optimization of a single parameter. An example will be given to show that very good designs can be obtained in spite of the limit of search.
\end{abstract}

Index Terms - Cosine-modulated filterbanks, filterbank design, Kaiser window.

\section{INTRODUCTION}

$\mathbf{T}$ THE THEORY and design of $M$-channel cosine modulated filterbanks have been studied extensively in the past (see, e.g., [1] for a list of references). The analysis and synthesis filters are cosine modulated versions of a lowpass prototype filter (as shown in Fig. 1). The design of the whole filterbank thus reduces to that of the prototype filter. The filterbank has perfect reconstruction if the polyphase components of the prototype satisfy some pairwise power complementary conditions [1]. The optimization of the prototype coefficients in this case is highly nonlinear. In an approximate reconstruction cosine modulated filterbank, aliasing is canceled approximately and the distortion is a delay only approximately. Suppose the prototype filter $P\left(e^{j \omega}\right)$ has linear phase. The conditions for approximate reconstruction can be stated in terms of $P\left(e^{j \omega}\right)$ as follows [1], [2]:

$$
\begin{aligned}
& \left|P\left(e^{j \omega}\right)\right| \approx 0, \text { for }|\omega|>\pi / M \\
& T\left(e^{j \omega}\right) \approx 1, \quad \text { where } \quad T\left(e^{j \omega}\right)=\sum_{k=0}^{2 M-1} \mid P\left(\left.e^{j(\omega-k \pi / M)}\right|^{2} .\right.
\end{aligned}
$$

The accuracy of the first approximation will in turn give a measure of the aliasing error, while the accuracy of the second approximation gives us a measure of the distortion error.

Traditional design approaches of the prototype usually involve nonlinear optimizations [3]. In [4], Creusere and Mitra proposed a very efficient prototype design method without using nonlinear optimizations. Instead of a full search, the search in [4] is limited to the class of filters obtained using the

Manuscript received December 24, 1997. This work was supported in part by the National Science Foundation under Grant MIP 0703755, and by Tektronix, Inc. The associate editor coordinating the review of this manuscript and approving it for publication was Prof. A. Tewfik.

Y.-P. Lin is with the Department of Electrical and Control Engineering, National Chiao Tung University, Hsinchu, Taiwan, R.O.C.

P. P. Vaidyanathan is with the Department of Electrical Engineering, California Institute of Technology, Pasadena, CA 91125 USA.

Publisher Item Identifier S 1070-9908(98)04338-7.

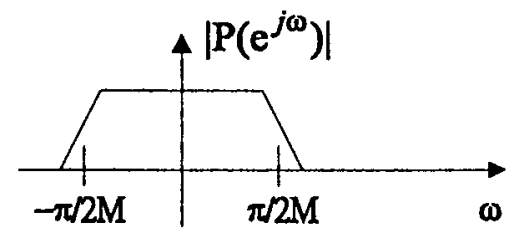

Fig. 1. Typical magnitude response of the prototype for an $M$-channel cosine modulated filterbank.

Parks-McClellan algorithm. As a result, the optimization can be reduced to that of a single parameter. The design examples provided therein have shown that very good prototypes that give close approximations of (1) and (2) can be obtained despite of the limit of search. Inspired by this, we show how the design method can be improved further by using the Kaiser window method.

In this letter, we consider limiting the search to the class of filters obtained using Kaiser window designs. Using this method, the optimization is also reduced to that of a single parameter. But the optimization procedure is even simpler than in [4] due to the fact that the filters designed by using a Kaiser window can be expressed in closed form. We will also propose the use of a new objective function that significantly reduces the amount of computation for the objective function. Experiments show that the objective function is a convex function of the parameter being optimized and that very good designs can be obtained. Furthermore, the resulting filterbank can be described by only four parameters regardless of the number of channels in the filterbank and the order of the prototype. This technique has the additional advantage that we have direct control over the stopband attenuation of the prototype filter and, hence, the stopband attenuation of the analysis and synthesis filters.

\section{Review of Prototype Designs Using THE PARKS-MCClellen AlgORITHM [4]}

In the design of a lowpass filter using the Parks-McClellan algorithm, there are four parameters to be determined, the passband edge $\omega_{p}$, the stopband edge $\omega_{s}$, passband-stopband error ratio $\gamma$, and filter length $N$. The first condition for approximate reconstruction (1) can be achieved by letting the stopband edge $\omega_{s}=\pi / M$. Now consider the second condition. Observing the expression of $T\left(e^{j \omega}\right)$ in (2) we see that $T\left(e^{j \omega}\right)$ is periodic with period $\pi / M$ and we only need to consider $T\left(e^{j \omega}\right)$ in the interval $[0, \pi / M)$. With $\omega_{s}=\pi / M$, we can 
approximate $T\left(e^{j \omega}\right)$ by

$$
\begin{gathered}
T\left(e^{j \omega}\right) \approx\left|P\left(e^{j \omega}\right)\right|^{2}+\mid P\left(\left.e^{j(\omega-\pi / M)}\right|^{2},\right. \\
\text { for } 0 \leq \omega<\pi / M . \\
\text { Let } \phi=\left.\max _{\omega}|| P\left(e^{j \omega}\right)\right|^{2}+\mid P\left(\left.e^{j(\omega-\pi / M)}\right|^{2}-1 \mid,\right. \\
0 \leq \omega<\pi / M .
\end{gathered}
$$

For a given filter length $N$ and an appropriately chosen passband-stopband error ratio $\gamma$, the passband edge $\omega_{p}$ is optimized to minimize $\phi$. Such a search reduces the design to only the optimization of the passband edge $\omega_{p}$. Experimentally, the objective function $\phi$ is shown to be a convex function of $\omega_{p}$ in the interval $[(\pi / 4 M),(\pi / M))[4]$.

\section{KAISER WINDOW APPROACH}

A filter $p(n)$ of length $N+1$ designed through Kaiser windows is of the form [5]

$$
p(n)=h_{i}(n) w(n)
$$

where

$$
h_{i}(n)=\frac{\sin \left(\omega_{c}(n-0.5 N)\right)}{\pi(n-0.5 N)}
$$

is the impulse response of the ideal filter with cutoff frequency $\omega_{c}$ and $w(n)$ is a Kaiser window. The window $w(n)$ is defined by

$$
w(n)= \begin{cases}\frac{\left.I_{o}(\beta) \sqrt{1-((n-0.5 N) / 0.5 N)^{2}}\right)}{I_{o}(\beta)}, & 0 \leq n \leq N \\ 0, & \text { otherwise }\end{cases}
$$

where $I_{0}(\cdot)$ is the zeroth-order modified Bessel function, which can be computed using

$$
I_{0}(x)=1+\sum_{k=1}^{\infty}\left(\frac{(0.5 x)^{k}}{k !}\right)^{2} .
$$

The parameter $\beta$ can be determined by the desired stopband attenuation [5]. So a filter designed using Kaiser windows can be specified by the three parameters $\omega_{c}, N$, and $\beta$.

Let us consider designing the prototype filter using Kaiser windows. Given a desired stopband attenuation $A_{s}$ and an appropriately chosen transition bandwidth $\Delta \omega$, the order of the window $N$ can be estimated by the following formula developed by Kaiser:

$$
N \approx \frac{A s-7.95}{14.36 \Delta \omega / 2 \pi}
$$

In this case, the window is completely determined. The cutoff frequency $\omega_{c}$ is the only parameter left in the Kaiser window design. Now we can optimize $\omega_{c}$ such that the objective function in (3) is minimized as in [4]. In what follows, we propose the use of a different objective function that is much easier to compute than that in (3).

Define a filter $G\left(e^{j \omega}\right)$ given by $G\left(e^{j \omega}\right)=\left|P\left(e^{j \omega}\right)\right|^{2}$. Observe that the condition in (2) means that $G\left(e^{j \omega}\right)$ is a

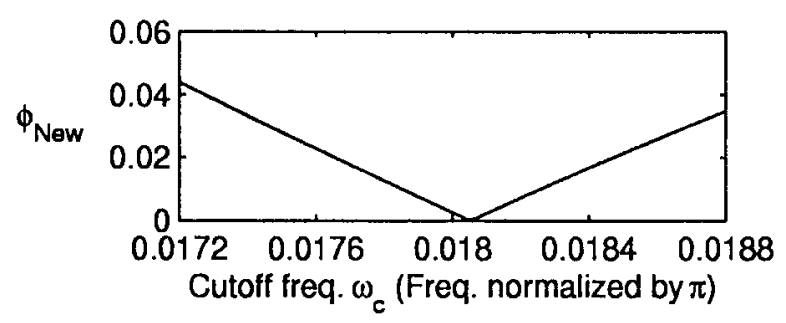

(a)

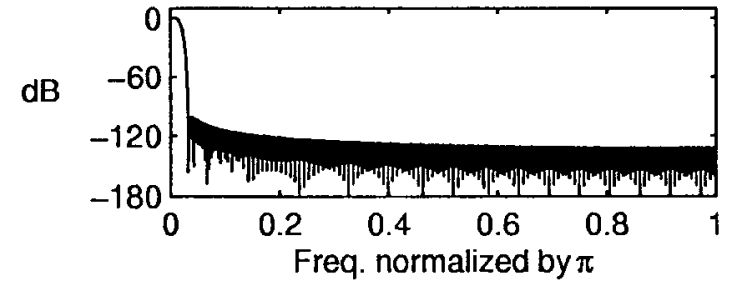

(b)

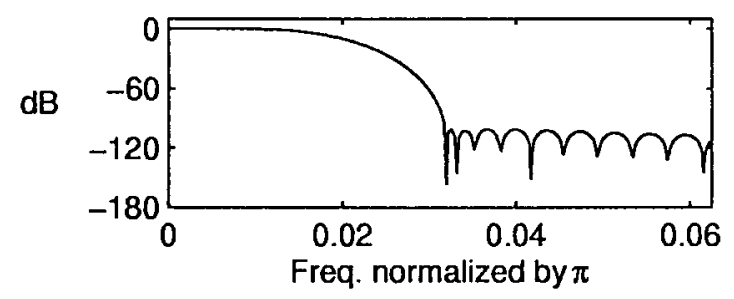

(c)

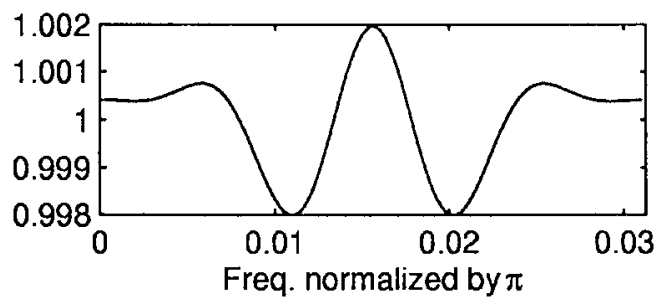

(d)

Fig. 2. (a) Objective function $\phi_{\text {new }}$ versus the cutoff frequency $\omega_{c}$. (b) Magnitude of $P_{0}\left(e^{j \omega}\right)$ (dB). (c) Zoom plot of (b) for the interval $[02 \pi / M]$. (d) Magnitude of $T\left(e^{j \omega}\right)$. As $T\left(e^{j \omega}\right)$ is periodic $\pi / M$, only the part in the interval $[0 \pi / M]$ is shown.

approximately a Nyquist $(2 M)$ filter. That is to say, $g(2 M n)$ is approximately $(1 / 2 M) \delta(n)$, where $\delta(n)$ is given by

$$
\delta(n)= \begin{cases}1, & n=0, \\ 0, & \text { otherwise. }\end{cases}
$$

Using this, we can choose a simpler objective function

$$
\phi_{\text {new }}=\max _{n, n \neq 0}|g(2 M n)| \text {. }
$$

We can adjust the parameter $\omega_{c}$ to find the best $p(n)$ that yields the smallest $\phi_{\text {new. }}$. Experiments show that $\phi_{\text {new }}$ is a convex function of $\omega_{c}$. Very good design can be obtained, as will be demonstrated in the next section.

\section{DESIGN EXAMPLE}

We use the proposed Kaiser window approach to design a cosine modulated filterbank with specifications similar to that of a Motion Pictures Expert Group (MPEG) audio coder. In 
this case, the filterbank has 32 channels and the prototype filter has stopband attenuation around $100 \mathrm{~dB}$ (pp. 397, [6]). So in the design we choose $A_{s}$ to be $100 \mathrm{~dB}$. The transition bandwidth is chosen to be slightly smaller than $\pi / 2 M$. The value of the transition bandwidth used in this example is $0.01406 \pi$. The order of the prototype $P\left(e^{j \omega}\right)$, estimated by (4), is 466. Fig. 2(a) gives the plot of the objective function $\phi_{\text {new }}$ versus the cutoff frequency $\omega_{c}$. From the plot we see that $\phi_{\text {new }}$ is a convex function of $\omega_{c}$; when $\omega_{c}=$ $0.01803 \pi$, the objective function $\phi_{\text {new }}$ has a minimum of $5.63 \times 10^{-4}$. Fig. 2 (b) shows the magnitude response (in $\mathrm{dB}$ ) of the optimized prototype filter. A zoom plot of Fig. 2(b) for the interval $[0,2 \pi / M]$ is given in Fig. 2(c). The magnitude response of the distortion function $T\left(e^{j \omega}\right)$ in this case is given in Fig. 2(d).

The design takes only a few seconds using a PC with a Pentium processor. ${ }^{1}$ A similar example using nonlinear optimizations will take approximately 5 min on a Sparc 10 workstation [3].

\section{CONCLUSIONS}

In this letter, we propose designing the prototype filters of cosine modulated filterbanks using Kaiser windows. This technique is simple conceptually and computationally. The design of the prototype filter is formulated as a problem of

\footnotetext{
${ }^{1}$ A Matlab program code of prototype designs using Kaiser windows is available at http://multirate.cn.nctu.edu.tw.
}

optimizing the cutoff frequency $\omega_{c}$ in Kaiser window designs. We further reduce the design complexity by introducing a new objective function $\phi_{\text {new }}$. Experiments show that $\phi_{\text {new }}$ is a convex function of $\omega_{c}$. We have demonstrated very good designs can be obtained, even when the desired stop attenuation is large and the filter order is high. Design examples have also shown that the design complexity of the Kaiser window approach is much less than that in nonlinear optimizations. On the other hand, because in Kaiser designs the stopband attenuation is determined by the window, we can have direct control over the stopband attenuation. Furthermore the coefficients of the prototype $p(n)$ can be expressed in closed form in terms of only three parameters, $\beta$, filter order $N$, and cutoff frequency $\omega_{c}$. So the whole cosine modulated filterbank can be specified by these three parameters and the number of channels $M$.

\section{REFERENCES}

[1] P. P. Vaidyanathan, Multirate Systems and Filter Banks. Englewood Cliffs, NJ: Prentice-Hall, 1993.

[2] J. H. Rothweiler, "Polyphase Quadrature Filters-A new subband coding technique," in Proc. IEEE Int. Conf. Acoustics, Speech, and Signal Processing, Boston, MA, Apr. 1983, pp. 1280-1283.

[3] G. Strang and T. Nguyen, Wavelets and Filter Banks. Wellesley, MA: Wellesley-Cambridge, 1996.

[4] C. D. Creusere and S. K. Mitra, "A simple method for designing highquality prototype filters for M-band pseudo QMF banks," IEEE Trans. Signal Processing, vol. 43, pp. 1005-1007, Apr. 1994.

[5] A. V. Oppenheim and R. W. Schafer, Discrete-Time Signal Processing. Englewood Cliffs, NJ: Prentice-Hall, 1989.

[6] M. A. Vetterli and J. Kovacevic, Wavelets and Subband Coding. Englewood Cliffs, NJ: Prentice-Hall, 1995. 